Proc. Indian Acad. Sci. (Earth Planet. Sci.), Vol. 95, No. 2, July 1986, pp. 201-208.

(C) Printed in India.

\title{
Effects of linear trend and mean value on maximum entropy spectral analysis
}

\author{
R P KANE and N B TRIVEDI \\ Instituto de Pesquisas Espaciais-INPE, Conselho Nacional de Desenvolvimento Científico e \\ Tecnológico-CNPq, $12200-S a$ José dos Campos, SP, Brasil
}

MS received 27 December 1984; revised 15 October 1985

\begin{abstract}
Using artificial samples, it is shown that, even for the method of maximum entropy spectral analysis (MESA) evolved by Burg, a presence of a linear trend gives distortions (frequency shifts) in the low frequency (high periodicity) region and hence, it is advisable to eliminate linear trends before attempting spectral analysis.

The effect of the presence of a non-zero mean is not very definite; but, very large mean values seem to reduce the sharpness of the peaks.

The implications of these findings for the results by Jin and Thomas for some geomagnetic parameters are discussed.
\end{abstract}

Keywords. Linear trend; maximum entropy spectral analysis; high periodicity; low frequency; geomagnetic parameters.

\section{Introduction}

For the study of periodicities in time series, Burg (1972) evolved the method of MEsA (maximum entropy spectral analysis) which is considered superior to earlier methods like those of Blackman and Tukey (1958). Ulrych and Bishop (1975) have given a critical appraisal of MESA.

There are some well-known drawbacks of MESA. Chen and Stegan (1974) showed that in cases where some large periodicities were involved, the spectral maxima may show frequency shifts (errors) as large as $20 \%$, depending upon the initial phase. Also, unlike the Blackman and Tukey method (1958) for which statistical significances can be estimated for the various observed peaks, there is no clear-cut method of estimating the statistical significance of MESA peaks.

In geophysical studies, spectral analysis is used frequently. Some geophysical parameters, like the geomagnetic field, have secular variations involving periodicities of several decades or sometimes just linear trends. Before subjecting such series to spectral analysis in search of smaller periodicities, it is recommended that long-term trends be removed (Bingham et al 1967) and many workers do so (e.g. Bhargava and Yacob 1970). However, recently, Jin and Thomas (1977) used MESA for studying the periodicities in the time-series of the geomagnetic dipole field $H_{0}(1901-1969)$ without removing the considerably large linear down-trend present in the data. We want to explore, with the help of artificial samples, whether the presence or absence of a non-zero mean and a linear trend affects the accuracy of the frequency determination. The drawbacks of MESA referred to above will be considered only to the extent that they affect conclusions about this specific aspect. Finally, the data used by Jin and Thomas (1977) will be subjected first to trend removal and later to MESA and the results compared with those obtained by them. 
It must be borne in mind that MESA is a nonlinear method for the spectral estimate. Hence, the elimination of trends and the mean may affect the spectral estimate. We hope to get some clues for this effect from the study of the artificial samples. In physical series, much will depend upon the physical models for a given time series.

\section{Analysis of artificial samples}

We produced an artificial time series $f(t)$ composed as:

$$
f(t)=a_{0}-a_{1} t+\sum_{k=1}^{5} b_{k} \sin 2 \pi\left(t / T_{k}\right)
$$

where $t$ ranged from 1 to 100 , the five sinusoids had periods $T_{k}=5,10,20,40,80$, the amplitudes $b_{k}$ were set at 10 for all $k$, and the constants $a_{0}$ and $a_{1}$ were varied. First, $a_{0}$ was set at 1000 and the slope $a_{1}$ was given values $a_{1}=0,12,24$ in turn. Thus, for the largest slope of $a_{1}=24$, the series started with a value of about 1050 and ended with a value of about - 1350. All the series were subjected to MESA, using the flow chart given by Anderson (1974).

The upper half of figure la shows the results for the length of the prediction error filter (LPEF) $=25,50,75,85,95$. In each case, the full curve refers to $a_{1}=0$, i.e. the sample with no linear trend, and the dashed curve refers to $a_{1}=24$. Curves for $a_{1}=12$ are not shown; but the three numbers one below the other represent the periodicities observed for $a_{1}=0,12,24$. The ordinate is power spectral density on a log scale. Usually, the power is calculated at fixed frequency intervals $\Delta f$ in the frequency range $f=0$ to 0.5 , the latter being the Nyquist (folding) frequency. However, in such a case, the fractional ratio $(\Delta / f)$ becomes non-uniform and is large for small frequencies and gives large steps for large periodicities. Hence, we chose frequencies corresponding to periodicities $T$ in steps of $\log _{10} T$ of 0.005 for the $\log _{10} T$ range of 0.300 to 2.300 corresponding to $T=2$ to 200 . This ensured that the steps were within about $\pm 1 \%$ of periodicity $T$ for all bands. In figure 1(a), the following may be noted:

(1) For LPEF $=25$ (i.e. $25 \%$ of the data length of 100 ), the resolution is poor. Only for LPEF of 50 or more, all the peaks are resolved, but only in the trendless sample (full lines). In the sample with linear trend (dashed lines), only the peaks at $T=5,10,20$ are resolved while those at $T=40$ and 80 are either missed or displaced, even at very high LPEF.

(2) At higher LPEF, the low periodicity peaks show splitting. This is a well-known feature and can be corrected for by using the modification formulated by Fougere (1977).

From the above example, it seems that the presence of a linear trend may cause considerable distortions in the low frequency (high periodicity) region.

For the above sample, $a_{0}$ was 1000 . To study the effect of large $a_{0}$ values, the value of $a_{0}$ was increased to 32000 . The two curves in the lower half of figure 1a refer to spectra of the samples with $a_{0}=32000$ and, $a_{1}=0$ (full curve) and $a_{1}=24$ (dashed curve). The peaks are now less sharp. However, whereas for $a_{0}=1000, a_{1}=24$, LPEF $=95$, the peaks at $T=40$ and $T=80$ were not resolved, for $a_{0}=32000, a_{1}=24, \mathrm{LPEF}=95$, the peaks are resolved. Thus, a very high initial value or mean seems to help in resolving the peaks in the presence of a linear trend. However, this needs further scrutiny. 


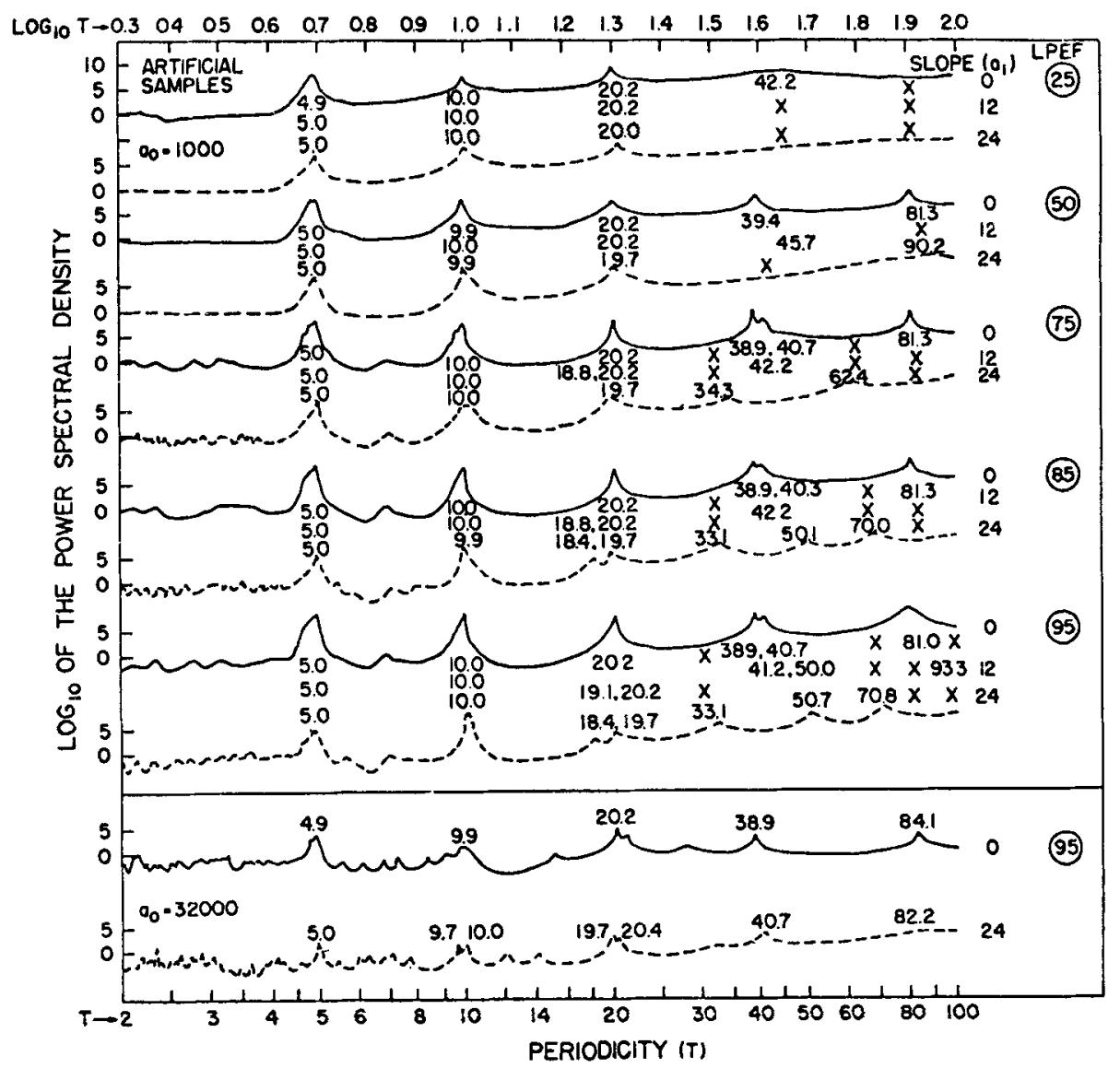

(a)

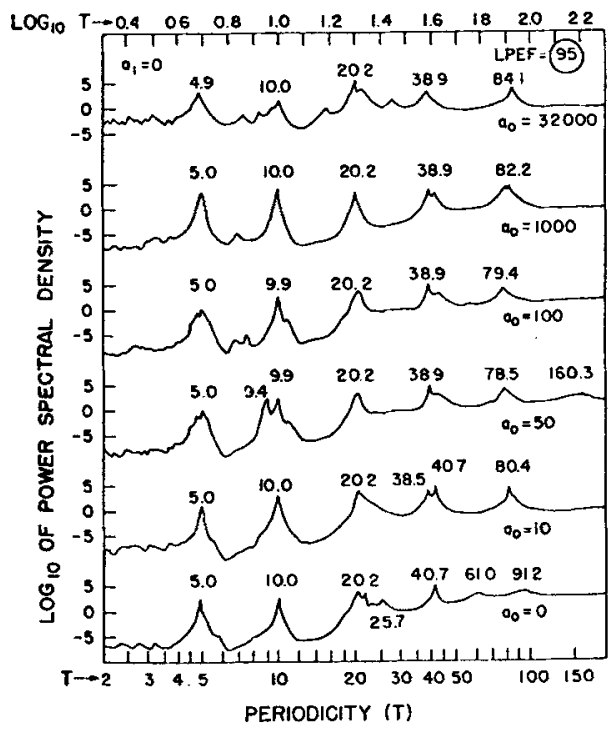

(b)
Figure 1. Burg spectra of artificial samples $\left(a_{0}\right.$ $-a_{1} t+$ sinusoids) of 100 data points ( $t=1$ to 100$)$ and five sinusoids of periods $T=5,10,20,40,80$ with, (a) linear down-trends of slopes $a_{1}=0$ (full lines) and $a_{1}=24$ (dashed lines). The set of three numbers one below the other represent peak periodicites for $a_{1}=0,12,24$. Value of $a_{0}=1000$ for all curves except the bottom two curves for which $a_{0}$ $=32000, \mathrm{LPEF}=95$ and, $a_{1}=0$ (full curve), $a_{1}=24$ (dashed curve); (b) no linear trends (i.e. $a_{1}=0$ ) but $a_{0}=32000,1000,500,100,50,0$, the last one representing a sample expressed as deviations from mean. $L P E F=95$ for all curves and numbers represent periodicities. 
To study the effect of $a_{0}$ values alone in greater detail and, in particular, to see whether a sample expressed as a deviation from the mean gave better results, we set $b_{k}=10$ as before, set $a_{1}=0$ (i.e. no linear trend) and set $a_{0}=0,10,50,100$ in turn. Figure $1 b$ shows the spectra for LPEF $=95$ only, for $a_{1}=0, b_{k}=10$ and $a_{0}=0,10,50$, 100,1000 and 32000 , the latter two being the same as the full curves at the bottom of figure la. As can be seen, all the peaks are resolved reasonably well in all the curves (but for some peak splitting) except the bottom curve for $a_{1}=0$ where the high periodicity peak $T=80$ is not resolved but splits into $T=61.0$ and $T=91 \cdot 2$. Thus, spectra of a sample expressed as deviations from mean may not depict the very high periodicity peaks correctly. On the other hand, adding even a small number as 10 , something of the same order as the amplitudes of the sinusoids, seems to help in resolving the very high periodicity peak, while adding too large a number (like $a_{0}=32000$ for the top curve) may distort the high periodicity peak by a few percent and reduce the sharpness of the peaks.

To test samples having linear trends only, i.e. having no sinusoids but only a down (or up) linear trend, we set $b_{k}=0$ and $a_{1}=25$ and $a_{0}=1250$ and 32000 . The former $\left(a_{0}\right.$ $=1250$ ) gives a sample having values ranging from +1250 to -1250 and thus having a mean zero. Figure 2 shows the spectra. Even though the sample contains no periodicities, the spectra show very low power for high frequencies only. For low frequencies, the power is comparatively large, indicating that a large linear trend could create distortions for periodicities in this region. The change from low power to high power is larger in the case of a lower mean $\left(a_{0}=1250\right.$, lower curve) and occurs at lower periodicities. Thus, analysis of data which are expressed as deviations from mean will probably have larger distortions due to the presence of linear trends as compared to the data with the same linear trend but larger base value $\left(a_{0}=32000\right.$, upper curve).

In the samples chosen so far, all sinusoids had the same amplitude $\left(b_{k}=10\right)$ and the periods formed a binary progression. To confirm that such a choice did not produce any special effect, we changed the amplitudes to $b_{k}=10,5,10,5,10$ and chose various sets of $T_{k}$ e.g. $T_{k}=6,10,16,36,76$ and many similar ones where the successive $T_{k}$ had no simple relationship. In all cases, results were similar to those mentioned above for $b_{k}=10$ and $T_{k}=5,10,20,40,80$.

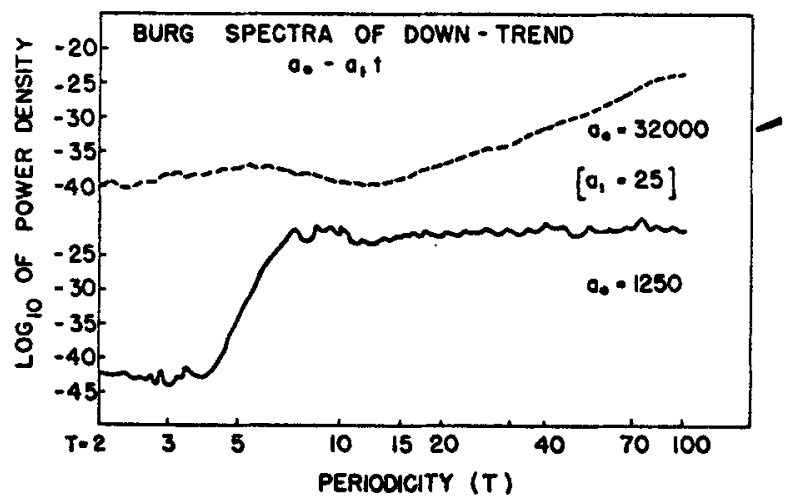

Figare 2. Burg spectra of pure linear trends $\left(a_{0}-a_{1} t\right)$ for $a_{1}=25$ and $a_{0}=32000$ (upper, dashed curve) and $a_{0}=1250$ (lower, full curve). 
We also tested whether, by removing some periods from the data by suitable filters, the residues showed the remaining periodicities in MESA. We found that all the periodicities which now existed were seen as sharp peaks in MESA. However, in all cases, the relative heights of the peaks were not in the same proportion as expected. This can be seen in figure 1 also where all peaks are not of the same height. But, there are no unwanted peaks either, at least in LPEF less than $50 \%$ of data length.

\section{Analysis of the geomagaetic field and other parameters}

From a spherical harmonic analysis of the annual mean magnetic data at various geomagnetic observatories round the globe, Jin and Thomas (1977) calculated the yearly Gauss-Schmidt coefficients and also $H_{0}$, the dipole field at the magnetic equator. Figure 3 (full curve) shows a plot of the yearly values of $H_{0}$ for 1901-69. From about $32300 n T$ in 1901, the value of $H_{0}$ dropped to about $30600 n T$ in 1969, a drop of about $1700 n T$ in 70 years i.e. about $24 n T /$ year. However, the values near 1901 seem to be abnormally large and those near 1969 abnormally low. When a first-degree polynomial (straight line) is fitted statistically, the dashed line in figure 3 has a form $H_{0}=32135$ $-(18 \cdot 2) t$, having a slope of only about $18 n T /$ year.

Figure 4 shows the spectra for $H_{0}$ (full lines) for LPEF $=25,50,60,65,68$ which is $36 \%, 72 \%, 87 \%, 95 \%, 98 \%$ of the data length 69 . For low LPEF, very few peaks are resolved. At very high LPEF, many peaks are resolved. The numbers represent periodicities in years. For LPEF $=68$ the numbers in boxes are those reported by Jin and Thomas (1977) and tally with our numbers, indicating that our method of spectral analysis is the same as used by them. The periodicities $T=66 \cdot 8,34 \cdot 3,22 \cdot 1,17 \cdot 8,13 \cdot 3$, $11 \cdot 1,9 \cdot 4,8 \cdot 5,7 \cdot 0$ do seem to be harmonics of a fundamental period $T=66.8$. However, when the linear trend is removed from the data, the Burg spectra are as shown by dashed lines in figure 4. For $\mathrm{LPEF}=68$, the peaks are now at $T=47 \cdot 3,28 \cdot 5,20 \cdot 2,16 \cdot 2$,

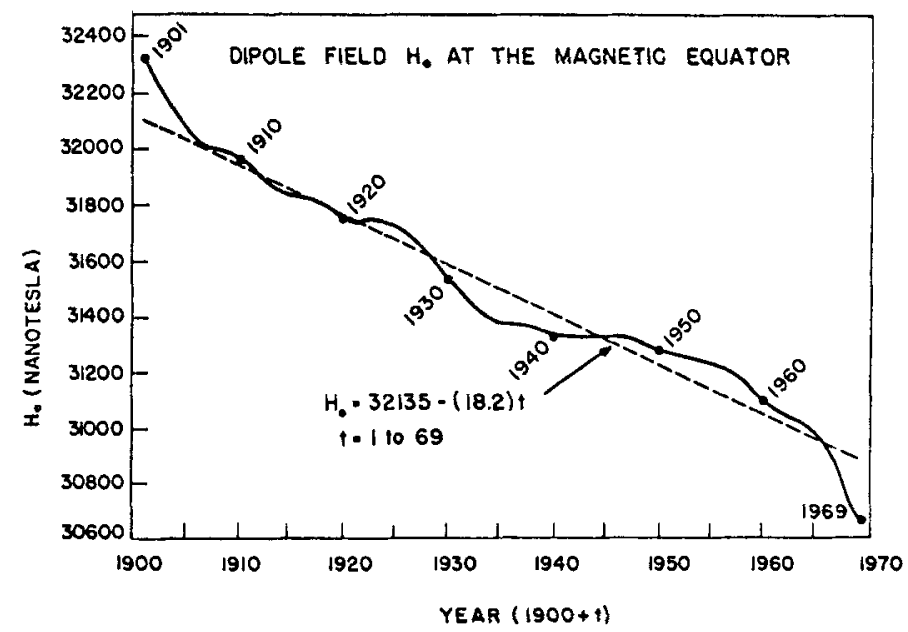

Figure 3. Time series of yearly means of dipole field $H_{0}$ at the magnetic equator for 1901-69 (Jin and Thomas 1977). The dashed line represents a statistical fit of a linear trend of slope about $18 n T /$ year. 


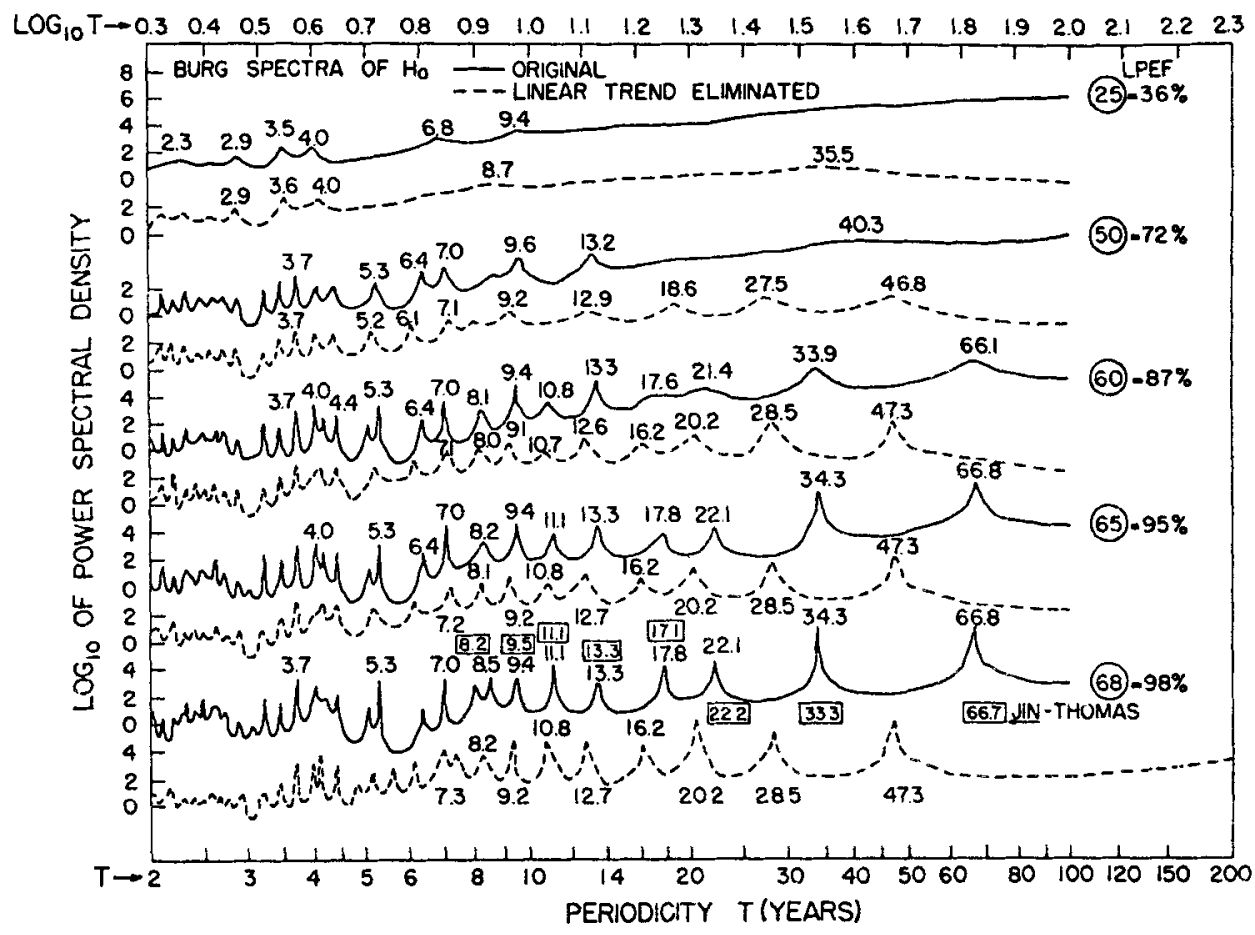

Figure 4. Burg spectra (full lines) of the 69 yearly values of geomagnetic dipole field $\boldsymbol{H}_{\mathbf{a}}$ for $L P E F=25,50,60,65,68$ i.e. $36,72,87,95,98 \%$ of the data length. Numbers represent periodicities in years. For $L P E F=68$, the numbers in boxes are the periodicities obtained by Jin and Thomas (1977). The dashed lines represent Burg spectra when a linear trend is statistically removed.

$12 \cdot 7,10 \cdot 8,9 \cdot 2,8 \cdot 2,7.3$ which seem to be odd harmonics (3rd, 5 th, 7 th etc.) of a fundamental period $T=142$ (though such a peak was not actually observed when analysis was extended beyond $T=100$ as shown in figure 4 , bottom curve). We do not propose to discuss the physical cause of these periodicities, as our purpose is only to study the distortion effects due to linear trends. It would seem that the answers obtained by Jin and Thomas, viz, $T=66.8,34.3$ etc. may not be the correct ones, which seem to be $T=47 \cdot 3,28 \cdot 5$ etc.

Jin and Thomas (1977) have reported results for two more physical parameters. One is the time rate of change of the geomagnetic dipole field $\left(\dot{H}_{0}\right)$ and the other is the fluctuations of the length of the day (l.o.d.). The plots of these parameters are shown in figures 5 and 6. Figure 5 refers to $\dot{H}_{0}$ for $1905-65$ and the 61 values are in the range $(0$ to -50$) n T /$ year with an average value of about $-25 n T /$ year. Figure 6 refers to l.o.d. for 1865-1961 and the 97 values are in the range +4 to -4 milliseconds, with an average value of about zero but with a long-term up-trend shown by the dashed line. For both these samples, Jin and Thomas (1977) have reported peaks similar to those for $H_{0}$, which led them to believe that all these have a common physical origin. We obtained spectra for the $\dot{H}_{0}$ and l.o.d. shown in figures 5 and 6 and obtained peaks similar to those reported by Jin and Thomas. However, when $H_{0}$ values were expressed as deviations from the mean of the sample, and l.o.d. values were corrected for the linear trend, the spectra looked as shown in figure 7. In the upper half, the full curve refers to 


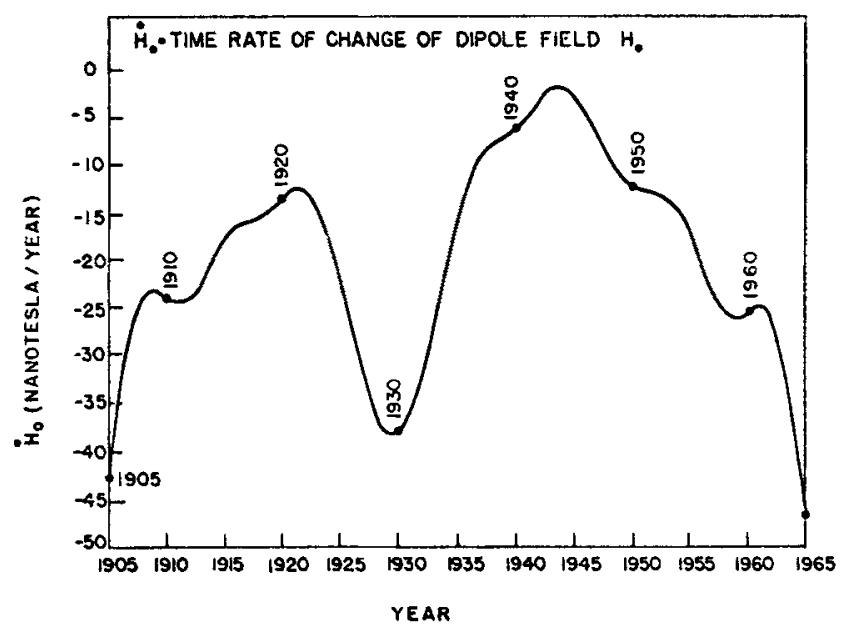

Figure 5. $\dot{H}_{0}$, the time rate of change of the geomagnetic dipole field $H_{0}$, for $1905-65$.

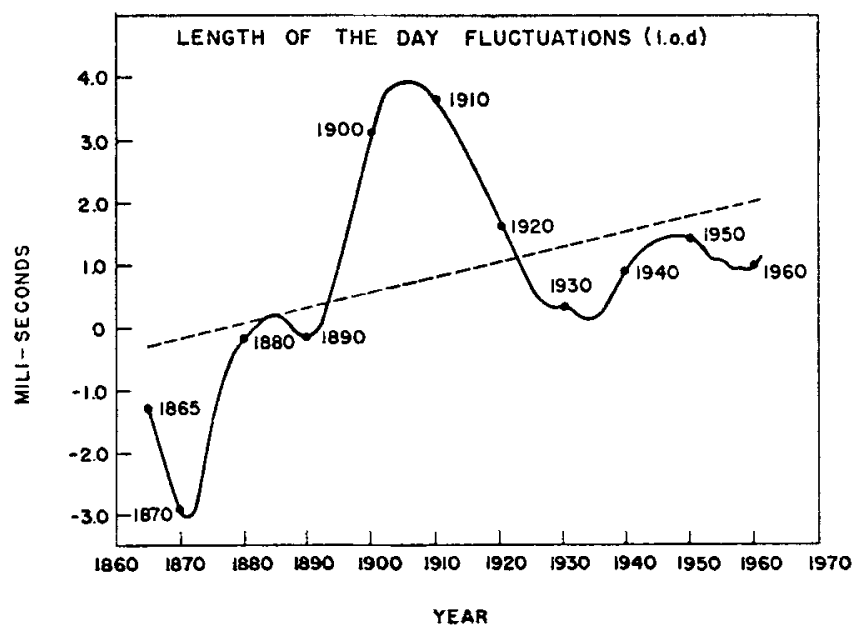

Figure 6. Fluctuations in the length of the day (1.o.d.) for 1865-1961. The dashed line is the statistical fit of a linear trend.

spectra of the original 61 values of $\dot{H}_{0}$, for an LPEF $=60$ i.e. $98 \%$, and the dashed curve refers to the values of $\dot{H}_{0}$ expressed as deviations from mean. The high-periodicity peak is at $T=65.3$ in one case and $T=69.2$ in the other. In the lower half of figure 7, the full curve represents the spectra of the original 97 values of $1.0 . d$. for an LPEF $=95$ i.e. $98 \%$ and the dashed is for l.o.d. values after eliminating the linear trend. The high-periodicity peak $T=65.3$ for the original values has been replaced by two peaks at $T=52.5$ and $T$ $=73 \cdot 3$. Thus, removal of a linear trend and/or a change of the mean value alter the results considerably in the high periodicity reoion. 


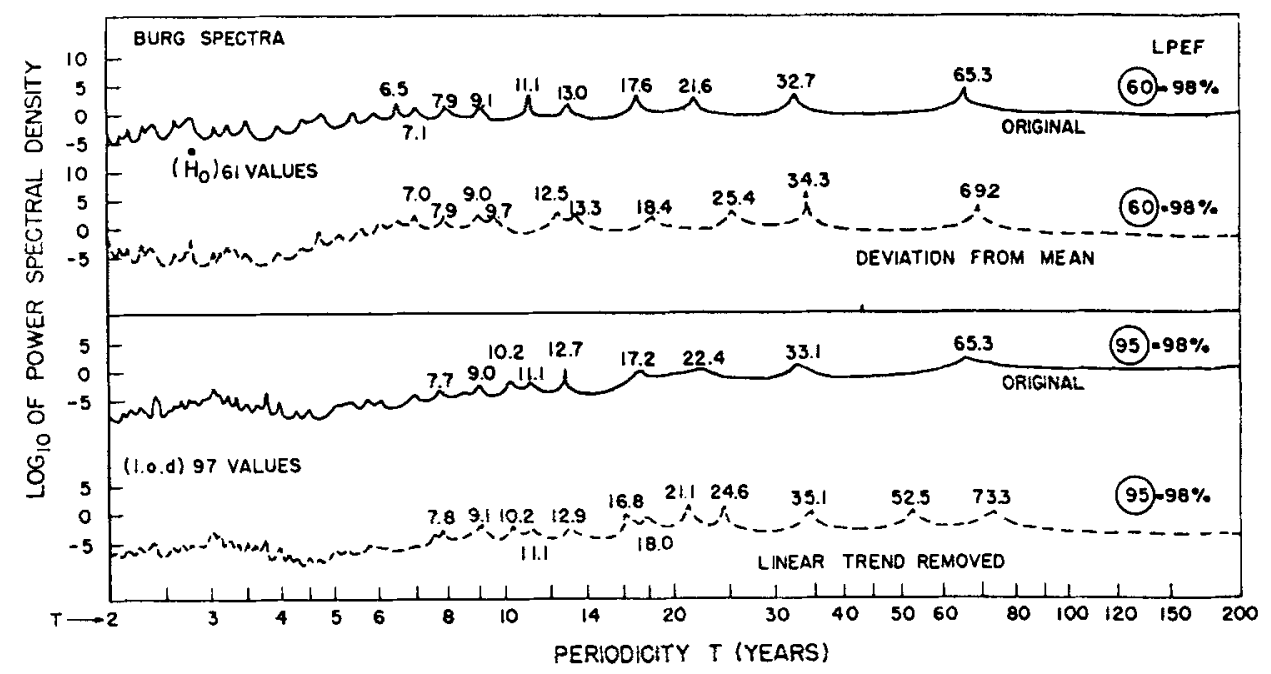

Figure 7. Burg spectra of $\dot{H}_{0}$, the time rate of change of $H_{0}(61$ values, upper half $)$ and of 1.o.d. fluctuations (97 values, lower half), for LPEF of about $98 \%$. For $\dot{H}_{0}$, the full curve is for original values and the dashed curve is for deviations from the mean of the whole sample. For 1.o.d., the full curve is for original values and the dashed curve is for values after eliminating linear trend. Numbers represent periodicities in years.

\section{Conclusion}

From an analysis of artificial samples, it seems that the presence of linear trends distorts the high periodicity peaks and hence it is advisable to eliminate linear trends, even when the sophisticated maximum entropy spectral analysis (MESA, evolved by Burg) is used. The effect of a very large, non-zero mean is to reduce the sharpness of the peaks, and to distort the very high periodicity peaks by a few percent. However, a sample expressed as deviation from mean does not necessarily give the best resolution. A nonzero mean, of the same order as the amplitudes of the sinusoids involved, seems to give better resolution of high periodicity peaks.

\section{Acknowledgements}

This work was partially supported by FNDCT, Brasil, under contract FINEP, 130/CT.

\section{References}

Anderson N 1974 Geophysics 3969

Bhargava B N and Yacob A 1970 J. Atmos. Terr. Phys. 32365

Bingham C, Godfrey M D and Tukey J W 1967 IEEE Trans. AU-15 56

Blackman R B and Tukey J W 1958 The measurement of power spectra (New York: Dover)

Burg J P 1972 Geophysics 37375 and references therein

Chen W Y and Stegan G R 1974 J. Geophys. Res. 793019

Fougere P F 1977 J. Geophys. Res. 821051

Jin R S and Thomas D M 1977 J. Geophys. Res. 82828

Ulrych T J and Bishop T N 1975 Rev. Geophys. Space Phys. 13183 